A - Research concept and design

B - Collection and/or assembly of data

$\mathrm{C}$ - Data analysis and interpretation

D - Writing the article

E - Critical revision of the article

F - Final approval of article

\section{Co-relation of pain, function, and quality of life with the radiographic findings in knee osteoarthritis patients}

\author{
Rekha Chaturvedi*A-F iD, Shabnam JoshiA,E-F iD \\ Guru Jambheshwar University of Science and Technology, India
}

*Correspondence: Rekha Chaturvedi; Guru Jambheshwar University of Science and Technology, India; email: rekhachaturvedi85@gmail.com

\begin{abstract}
Introduction: Osteoarthritis (OA) is a prevalent musculoskeletal disorder. In the diagnosis of OA, both the radiographic and clinical findings are used however, various inconsistencies in these systems generate a need to explore to which extent these systems should be followed to establish a diagnosis about OA. Thus, the objective of the study is to find out the co-relation of pain, function, and quality of life with the radiographic findings in patients with knee osteoarthritis.

Material and methods: Study design: Multi-center prospective cross-sectional study. 83 patients were screened and 72 patients were enrolled in the study following the inclusion and exclusion criteria. For radiographic evaluation, Kellgren Lawrence grading system, for pain, visual analog scale (VAS) and the function was measured by six-minute walk test and disability and quality of life was measured by Knee injury and osteoarthritis outcome score (KOOS). Statistical analysis was done using the Kendall tau test.

Results: The result showed significant co-relation of pain (VAS) with the radiographic findings, whereas a significant negative co-relation of knee osteoarthritis outcome score (KOOS) sub-variables of symptom, pain, function, and sports function with the radiographic findings. No significant co-relation in the six-minute walk test and KOOS quality of life sub-variable was found with the radiographic findings in knee OA patients.

Conclusions: Pain is significantly co-related with the radiographic findings. However, the functions were not significantly correlated with the radiographic findings in patients with knee osteoarthritis. Therefore, in addition to radiographic changes, the function of the patients must be assessed to estimate the progression of the disease.
\end{abstract}

Keywords: joint diseases, visual analog scale, walk test, X-Rays

\section{Introduction}

Osteoarthritis (OA) is amongst the highly prevalent musculoskeletal disorders. According to Global burden of diseases 2018, it estimates to affect 330 million people globally [1]. The prevalence of OA is on an exponential rise because of the surge in ageing population and an increase in obesity profiles and increased rates of traumatic knee injuries [2,3]. The prevalence of OA is seen to increase with the increase in the age with female gender having a higher preponderance to develop $\mathrm{OA}$ as compared to males [4]. Knee osteoarthritis is prevalent in Asian countries because of certain lifestyle habits that result in greater loads at the weight bearing 
joints [5]. The common symptoms of knee osteoarthritis (KOA) include pain, joint stiffness, decrease in range of motion, swelling, crepitus, locking and sensation of giving way of the joint [6].

For establishing a diagnosis regarding the disease, both the subjective as well as objective assessment is followed. Radiographic and clinical findings of KOA patients are compared to establish an accurate diagnosis of the condition. Various radiographic scales such as Kellgren Lawrence (KL) score system, Knee Society Radiographic Scoring System and criteria laid by American College of Rheumatology (ACR) for clinical diagnosis are commonly used for diagnosing the severity of OA. However, because of the various inconsistencies in these systems there is a need to explore to which extent these systems should be followed to establish a diagnosis about OA. A study has shown that the radiographic findings of tibiofemoral joint are co-related to a greater extent as compared to patellofemoral joint in specific clinical findings A study has shown moderate co-relation of clinical results with the radiographic findings for KOA diagnosis [7]. Whereas, one study reports that the symptoms such as pain and stiffness prominently affects the functional status in KOA patients and should be focused over the radiographic findings in patients of knee osteoarthritis to estimate the progression of the condition [8].

Thus, understanding the relationship of radiographic findings with the clinical symptoms must be explored further as, this is of utmost importance in the making clinical decision regarding knee OA patients. The purpose of the study is to find out the relationship of pain, function, disability and quality of life with the radiographic findings in KOA patients.

\section{Materials and methods}

The present study is a multi-center prospective cross-sectional study. Patients diagnosed with KOA as per ACR criteria were enrolled in the study following the inclusion and exclusion criteria [9]. Patients diagnosed with KOA, able to read and write English, Hindi and Urdu language and willing to participate were included in this study. The patients were excluded if they had history of knee injury, inflammatory arthropathies, metabolic bone diseases, were unable to walk for $6 \mathrm{mi}$ nute, unable to walk unaided and intraarticular injection in last 6 months. Eighty three patients with knee OA were screened for participation in the study. Out of these, eleven patients were excluded, three patients had history of intra-articular injection, five had history of injury to the knee joint, one had inflammatory arthritis and two patients were unable to walk unaided. Seventy two patients were included to participate in the study. Ethical approval was obtained from Institutional ethical committee vide letter no. PTY/2017/484 dated $9^{\text {th }}$ October 2017 and was done in accordance with the declaration of Helsinki, 2013. A written informed consent was obtained from all the participants prior to participation in the study. The sample size estimation was done using G power at $80 \%$ power and $95 \%$ significance level and the result suggested of a total of 64 patients.

\section{Procedure}

The patients were evaluated at baseline day and demographic data was collected. Visual Analogue Scale (VAS) was used to measure pain during walking a short distance. The pain was assessed on a $10 \mathrm{~cm}$ scale where 0 indicates no pain and 10 indicate severe pain. For radiographic evaluation, the radiographs were taken at weight bearing position from the Antero-posterior side. The radiographic evaluation was done using Kellgren Lawrence Grading Scale (KL) [10]. KL system involves measuring the changes in the joint seen on the radiographs with grades from $0-4$, where 0 denotes minimal changes or near normal radiographs, 1 denotes doubtful pathology, 2 denotes presence of minimal osteophytes, possible narrowing of the joints, presence of cysts and sclerosis, 3 denotes moderate changes and presence of definite osteophytes with moderate joint space narrowing and 4 denotes severe changes with presence of large osteophytes and definite joint space narrowing. The radiographs were evaluated by an experienced orthopedician who referred patients for the study. For assessment of function six minute walk test was used. The patient was asked to walk between the $30 \mathrm{~m}$ corridor which was marked by cones with no verbal feedback on the pace of walking. Sixminute walk test is a reliable tool for objective measurement of function in knee osteoarthritis [11]. The disability was measured using Knee Osteoarthritis Outcome Score (KOOS) questionnaire which is an extension of WOMAC and contains symptoms, pain, function, sports and recreation and quality of life components. The score ranges from 0 to 100 where 100 indicate a minimum problem. KOOS is a reliable assessment tool in Indian knee OA patients $[12,13]$.

\section{Statistical analysis}

The data was reported as mean and standard deviation and was analyzed using SPSS version 25.0. The significance level was compared at $\mathrm{p}<.05$. The Kendall tau was used to determine co-relation between the radiographic findings and pain, function and disability. Kendall tau is a non parametric test to estimate correlation between variables for small sample size. 


\section{Results}

Table 1 shows the demographic characteristics of the participants included in the study. A total of 72 patients (21 males and 51 females) diagnosed with knee OA were included in the study. Left knee was affected in 28 patients and right knee was affected in 22 patients, while 20 patients had bilateral KOA. KL grading was done for all the patients and $43.8 \%$ patients had grade 3 , $31.5 \%$ patients had grade $2,3.4 \%$ had grade 1 and $2.2 \%$ had grade 4 as per Kellgren Lawrence grading system for radiographic evaluation of the condition.

Tab.1. The demographic feature, clinical and radiological characteristics of the participants included in the study

\begin{tabular}{lc}
\hline Variables & $\mathrm{N}=72$ \\
\hline Age $($ years $)[$ Mean $\pm \mathrm{SD}]$ & $53.58 \pm 5.99$ \\
Height $(\mathrm{cm})[\mathrm{Mean} \pm \mathrm{SD}]$ & $159.02 \pm 7.36$ \\
Weight $(\mathrm{kg})[$ Mean $\pm \mathrm{SD}]$ & $72.16 \pm 9.50$ \\
BMI $\left(\mathrm{Kg} / \mathrm{m}^{2}\right)[\mathrm{Mean} \pm \mathrm{SD}]$ & $28.62 \pm 3.97$ \\
Affected Knee $(\mathrm{Left})$ & $28(38.8 \%)$ \\
Affected Knee $(\mathrm{Right})$ & $22(30.5 \%)$ \\
Bilateral Knee OA & $20(27.7 \%)$ \\
Males & $20(28.2 \%)$ \\
Females & $48(67.6 \%)$ \\
KL grade 1 & $3(3.4 \%)$ \\
KL grade 2 & $28(31.5 \%)$ \\
KL grade 3 & $39(43.8 \%)$ \\
KL grade 4 & $2(2.2 \%)$ \\
\hline
\end{tabular}

$\mathrm{KL}=$ Kellgren Lawrence grading

Tab. 2. The values of the Co-relation between the variables and radiographic findings

\begin{tabular}{lcc}
\hline Variables & $\begin{array}{c}\text { Co-relation } \\
\text { coefficient }(\mathrm{r})\end{array}$ & $\mathrm{p}$ value \\
\hline Age & $0.316^{* *}$ & 0.001 \\
Weight & $0.357^{* *}$ & 0.000 \\
BMI & $0.231^{*}$ & 0.016 \\
VAS & $0.240^{*}$ & 0.021 \\
6MWT & -0.140 & 0.147 \\
KOOS Symptom & $-0.264^{*}$ & 0.008 \\
KOOS Pain & $-0.229^{*}$ & 0.021 \\
KOOS Function & $-0.321^{*}$ & 0.001 \\
KOOS Sports Function & $-0.249^{*}$ & 0.012 \\
KOOS Quality of life & -0.065 & 0.526 \\
\hline
\end{tabular}

** Significant at $\mathrm{p}<0.001, *$ significant at $\mathrm{p}<0.05$. VAS $=\mathrm{Vi}-$ sual analog scale, $6 \mathrm{MWT}=$ Six minute walk test, $\mathrm{KOOS}=$ Knee injury and osteoarthritis outcome score.
Table 2 shows the value of correlation between the variables and the radiographic findings. The results of the study showed a significant co-relation of age with the KL scoring, weight and BMI with the radiographic findings. There was a significant co-relation of pain and KL scoring. However, no significant co-relation between function measured by six minute walk test and the radiographic findings was observed. The disability and quality of life measured using KOOS showed significant negative co-relation in symptom, pain, function and sports function sub-variable and no significant corelation was found in quality of life sub variable with the radiographic findings.

\section{Discussion}

Pain is a salient feature of KOA. The presence of knee pain is significantly affects the function and contributes enormously to disability and the risk of disability increases further with the presence of knee pain in the community $[14,19]$. A study has shown that OA is amongst the most highly prevalent musculoskeletal condition and is amongst the chief contributor to global YLD and contributes $2.4 \%$ of all years lived with disability (YLD) and has been ranked $10^{\text {th }}$ in contributing to global YLD's and imposes a huge socioeconomic burden to the society $[15,16]$. For making a diagnosis about the condition conventional radiographs are commonly used. Various other methods are also available that classifies the specific features of OA but are less popular and have low validity and sensitivity. Thus, radiographs being easily available, economical and well accepted by the patients are considered as cornerstone in diagnosing KOA $[17,18]$.

However, there is discordance in the available literature on the importance of radiographs in diagnosing the condition. But radiographs are often used by the surgeons in making judgment regarding surgical procedures such as arthroplasty. Thus, it is very important to understand the relationship of pain, function, disability and quality of life with the radiographic findings in KOA patients. So, the present study aimed at estimating the co-relation of pain, function and quality of life with the radiographic findings in knee OA patients.

The result of the study showed a significant co-relation of pain with the radiographic findings. However, there was no significant co-relation of function measured by six minute walk test and quality of life with the radiographic findings. The significant correlation of pain found in the present study was consistent with the findings of various studies that showed a strong association of radiographic osteoarthritis with the knee pain $[19,20]$. Another study has also demonstrated that the 
knee pain was significantly associated the presence of definite KOA with KL grading more than or equal to grade 2 and with the presence of all individual features [21]. In consistence the present study also had greater number of patients in grade $2(31.5 \%)$ and grade 3 $(43.8 \%)$ of the KL scoring reflecting significant correlation of pain with the radiographic findings. Similarly, some other studies have also shown that individuals that have presence of both the osteophytes and joint space narrowing i.e KL grade 3 or higher had highest odds of having knee pain [22,23].

The significant co-relation of pain with the radiographic findings can also be because of the greater number of female participants in the study. Various studies have also reported greater incidences of symptomatic $\mathrm{KOA}$ in females as compared to males [4,24]. Boyan et al. has suggested three possible causes for the gender differences as biomechanical differences in limb alignment, obliterated protective effect of estrogen after menopause and neurologic contribution for increased disease severity is seen in females being responsible for higher preponderance of OA in females [25].

Cubukcu et al. has also shown that the severity of knee pain is more important feature in determining the disability than the radiographic findings [8]. The result of the present study showed no significant correlation of function measured by six minute walk test and quality of life with the radiographic findings. Various studies have shown that the function has a strong relationship with the structural changes in early disease [26,27] Creamer et al. has also shown that pain and obesity are more important determinant in estimating the function in KOA as compared to the changes in the structure seen on radiographs, as the radiographs only shows the bony changes while muscles and ligament are important determinants of the functions in patients with KOA [26]. Similarly, Larson et al. reported that the functional ability was not related to radiographic diagnosis of osteoarthritis [28]. Cubukcu et al. have also reported to have no correlation between function and radiographic features [8]. It was also reported that obesity is another major determinant of functional loss and is associated with voluntary weakness of quadriceps muscle in patients with knee OA [29].

In addition to this, the present study has also found significant correlation of age, height, weight and BMI with the radiographic findings. Thus, it can be interpreted that the pain and symptoms can be reflected by the structural changes seen on radiographs but in order to accurately estimate about the condition, assessment of function should be done separately. As various factors such as strength of muscle, psychological status of patient, lifestyle habits and education about the condition might have influence on the functional ability of the patient [30]. The negative correlation of KOOS sub variables and no correlation of function measured by six minute walk test and quality of life with the radiographic findings reflect that the structural changes seen on radiographs does not predict about the function and quality of life of OA patients, thus interventions such as exercises and physiotherapy management can prove to be beneficial in improving the functions and activities of daily living as well as quality of life and can help in decreasing the requirement of operative and corrective procedures required in patients with KOA. The strength of the present study is that the subjective, objective as well as radiographic evaluation was done to estimate the co-relation between the variables. Loss of longitudinal follow up, small number of patients and confounding variables such as presence of co-morbidities like diabetes, hypertension were some potential limitations of the study.

Therefore, it is important to refer the radiographs along with the clinical findings to make an accurate diagnosis of the condition and most importantly functional assessment mimicking the activities of daily living should also be done to accurately estimate the progression of the disease. It is also suggested that newer methods that includes both the radiographic findings as well as objective assessment such as "New Knee Society Scoring system" should be adopted in diagnosing OA in place of the conventional system. Therefore, understanding the relationship of these variables can be of utmost importance in formulating an accurate diagnosis and estimating the progression of disease.

\section{Conclusion}

It can be concluded that the radiographic changes must be given importance in formulating a diagnosis of KOA. However, symptoms such as pain and stiffness along with the estimation of functional status of the patients must be done in addition to accurately interpret about the disease status, as this can play a vital role in clinical decision making process for patients affected with KOA.

\section{References}

1. James SL, Abate D, Abate KH, Abay SM, Abbafati C, Abbasi N, et al. Global, regional, and national incidence, prevalence, and years lived with disability for 354 diseases and injuries for 195 countries and territories, 1990-2017: a systematic analysis for the Global Burden of Disease Study 2017. Lancet. 2018; 392(10159): $1789-858$. 
2. Ogden CL, Carroll MD, Lawman HG, et al. Trends in Obesity Prevalence Among Children and Adolescents in the United States, 1988-1994 Through 20132014. JAMA. 2016; 315(21): 2292-9.

3. Lohmander LS, Englund PM, Dahl LL, Roos EM. The long-term consequence of anterior cruciate ligament and meniscus injuries: osteoarthritis. Am J Sports Med. 2007; 35(10): 1756-69.

4. Pal CP, Singh P, Chaturvedi S, Pruthi KK, Vij A. Epidemiology of knee osteoarthritis in India and related factors. Indian J Orthop. 2016; 50(5): 518-22.

5. Fransen M, Bridgett L, March L, Hoy D, Penserga E, Brooks P. The epidemiology of osteoarthritis in Asia. Int J Rheum Dis. 2011; 14(2): 113-21.

6. Hunter DJ, McDougall JJ, Keefe FJ. The symptoms of osteoarthritis and the genesis of pain. Rheum Dis Clin North Am. 2008; 34(3): 623-43.

7. Parsons C, Fuggle NR, Edwards MH, et al. Concordance between clinical and radiographic evaluations of knee osteoarthritis. Aging Clin Exp Res. 2018; 30(1): 17-25.

8. Cubukcu D, Sarsan A, Alkan H. Relationships between Pain, Function and Radiographic Findings in Osteoarthritis of the Knee: A Cross-Sectional Study. Arthritis. 2012, 984060.

9. Altman R, Asch E, Bloch D, et al. Development of criteria for the classification and reporting of osteoarthritis. Classification of osteoarthritis of the knee. Diagnostic and Therapeutic Criteria Committee of the American Rheumatism Association. Arthritis Rheum. 1986; 29(8): 1039-49.

10. Kohn MD, Sassoon AA, Fernando ND. Classifications in Brief: Kellgren-Lawrence Classification of Osteoarthritis. Clin Orthop Relat Res. 2016; 474(8): 1886-93.

11. Ateef M, Kulandaivelan S, Tahseen S. Test-retest reliability and correlates of 6-minute walk test in patients with primary osteoarthritis of knees. Indian J Rheumatol. 2016; 11: 192-6.

12. Jha RK, Sen RK, Tripathy SK, Gopinathan NR, Goyal T, Sharma SK. Cross-cultural validation of Hindi version Knee Injury and Osteoarthritis Outcome Score (KOOS) in osteoarthritis knee. Knee Surg Sports Traumatol Arthrosc. 2021 Jun; 29(6): 1742-9.

13. Ateef M, Kulandaivelan S, Alqahtani M. Cross-cultural validation of Urdu version KOOS in Indian population with primary knee osteoarthritis. Int J Rheumatol. 2017; 1206706.

14. Torres L, Dunlop DD, Peterfy C, et al. The relationship between specific tissue lesions and pain severity in persons with knee osteoarthritis. Osteoarthritis Cartilage. 2006; 14(10): 1033-40.

15. Vos T, Barber R, Bell B, Bertozzi-Villa A, Biryukov $\mathrm{S}$, Bolliger I, et al. Global, regional, and national incidence, prevalence, and years lived with disability for 301 acute and chronic diseases and injuries in 188 countries, 1990-2013: a systematic analysis for the Global Burden of Disease Study 2013. Lancet. 2015; 386(9995): 743-800.

16. Kotlarz H, Gunnarsson CL, Fang H, Rizzo JA. Insurer and out-of-pocket costs of osteoarthritis in the US: evidence from national survey data. Arthritis Rheum. 2009; 60(12): 3546-53.

17. Wang X, Oo WM, Linklater JM. What is the role of imaging in the clinical diagnosis of osteoarthritis and disease management? Rheumatology (Oxford). 2018; 57(suppl_4): iv51-iv60.

18. Wang K, Kim HA, Felson DT, et al. Radiographic Knee Osteoarthritis and Knee Pain: Cross-sectional study from Five Different Racial/Ethnic Populations. Sci Rep. 2018; 8, 1364.

19. Özden F, Nadiye Karaman Ö, Tuğay N, et al. The relationship of radiographic findings with pain, function, and quality of life in patients with knee osteoarthritis. J Clin Orthop Trauma. 2020 Jul; 11(Suppl 4): S512-S517.

20. Lethbridge-Cejku M, Scott WW Jr, Reichle R, et al. Association of radiographic features of osteoarthritis of the knee with knee pain: data from the Baltimore Longitudinal Study of Aging. Arthritis Care Res. 1995; 8(3): 182-8.

21. Guccione AA, Felson DT, Anderson JJ. Defining arthritis and measuring functional status in elders: methodological issues in the study of disease and physical disability. Am J Public Health. 1990; 80(8): 945-9.

22. Lawrence JS, Bremner JM, Bier F. Osteo-arthrosis. Prevalence in the population and relationship between symptoms and x-ray changes. Ann Rheum Dis. 1966; 25(1): 1-24.

23. Spector TD, Hart DJ, Byrne J, Harris PA, Dacre JE, Doyle DV. Definition of osteoarthritis of the knee for epidemiological studies. Ann Rheum Dis. 1993; 52(11): 790-4.

24. Cushnaghan J, Dieppe P. Study of 500 patients with limb joint osteoarthritis. I. Analysis by age, sex, and distribution of symptomatic joint sites. Ann Rheum Dis. 1991;50(1): 8-13.

25. Boyan BD, Tosi LL, Coutts RD, et al. Addressing the gaps: sex differences in osteoarthritis of the knee. Biol Sex Differ. 2013; 4(1): 4.

26. Creamer P, Lethbridge-Cejku M, Hochberg MC. Factors associated with functional impairment in symptomatic knee osteoarthritis. Rheumatology (Oxford). 2000; 39(5): 490-6.

27. Van Baar ME, Dekker J, Lemmens J, Oostendorp RA, Bijlsma J. Pain and disability in patients with 
osteoarthritis of hip or knee: the relationship with articular, kinesiological and psychological variables. J Rheumatol. 1998; 25: 125-33.

28. Larsson AC, Petersson I, Ekdahl C. Functional capacity and early radiographic osteoarthritis in middle-aged people with chronic knee pain. Physiother Res Int. 1998; 3(3): 153-63.
29. Slemenda C, Brandt KD, Heilman DK, et al. Quadriceps weakness and osteoarthritis of the knee. Ann Intern Med. 1997; 127(2): 97-104.

30. Fernandes L, Hagen KB, Bijlsma JW, et al. EULAR recommendations for the non-pharmacological core management of hip and knee osteoarthritis. Ann Rheum Dis. 2013; 72(7): 1125-35. 